\title{
A IMPORTÂNCIA DA GESTÃo DE PESSOAS NO AGRONEGÓCIO
}

\author{
THE IMPORTANCE OF PEOPLE MANAGEMENT IN AGRIBUSINESS
}

Lavínia Gabriele da Silva - lavinia-gabriele1998@ hotmail.com Faculdade de Tecnologia de Taquaritinga (FATEC) -SP -Brasil

José Guilherme Pandolfi - jgpandolfi@gmail.com

UNESP - Jaboticabal - SP - Brasil

Marcos Alberto Claudio Pandolfi - marcos.pandolfi@ fatectq.edu.br

Faculdade de Tecnologia de Taquaritinga (FATEC) -SP -Brasil

DOI: 10.31510/infa.v16i2.676

\section{RESUMO}

Este trabalho teve como objetivo apresentar a importância da gestão de pessoas no setor do agronegócio onde o seu papel é imprescindível dentro das organizações, devido ao conjunto de práticas que objetivam adequação dos indivíduos ao ambiente de trabalho para a realização das atividades. Para isso esse estudo teve como base a revisão bibliográfica com o objetivo de identificar quais são as práticas de gestão de pessoas utilizadas pelos agricultores, durante o recrutamento e seleção, treinamento e desenvolvimento, motivação e remuneração. Neste sentido, a pesquisa aponta que a solução depende dos procedimentos do gestor durante o processo de decisão, cabendo a ele enxergar um novo modelo de gestão, se adequando e estabelecendo critérios que possam aproveitar o potencial de cada pessoa, a fim de se obter melhores resultados para a empresa. Com as novas tecnologias chegando ao mercado, com o intuito de melhorar a produção o produtor ou proprietário tem que estar preparado com funcionários qualificados para atender essa demanda.

Palavras-chave: Gestão de pessoas. Agronegócio. Recrutamento e seleção.

\begin{abstract}
This work aims to present the importance of people management in the agribusiness sector, where its role is indispensable within organizations, due to the set of practices that aim to adapt the use of the work environment to activities. For this, this study was based on a literature review to identify the people management practices used by farmers during recruitment and selection, training and development, motivation and remuneration. In this sense, the research points out that the solution depends on the manager's procedures during the decision process. It is up to him to see a new management model, adapting and establishing criteria that can take advantage of each person's potential, in order to obtain better results for the company. With new technologies coming to the market, in order to improve production the producer or owner has to be prepared with qualified employees to meet this demand.
\end{abstract}

Keywords: People management. Agribusiness. Recruitment and selection. 


\section{INTRODUÇÃO}

Segundo Deadrick e Stone (2014), o desenvolvimento da gestão de pessoas teve seu início na Idade Média por meio do sistema de contratação do período medieval e veio evoluindo constantemente. Nota-se que as práticas de gestão de pessoas estão presentes em vários setores, contudo, nem sempre é possível caracterizar de que modo são utilizadas.

Essas práticas buscam solucionar os desafios de competitividade relacionados às mudanças de mercado, globalização, tecnologia, contenção de custos e melhor alocação dos funcionários. Para que isso ocorra é necessário desenvolver as atividades de planejamento, recrutamento, seleção, treinamento, avaliação, comunicação, remuneração e relações trabalhistas (BOHLANDER; SNELL, 2015).

As práticas de gestão de pessoas vêm ganhando espaço no setor agrícola, pois as máquinas realizam um trabalho eficiente, mas a participação humana é essencial no processo produtivo, como são as pessoas que controlam as máquinas, elas precisam estar motivadas a encontrar bons resultados, e treinadas para exercer sua função de forma eficiente.

O objetivo do agronegócio é aumentar a produtividade através da introdução de novas tecnologias, nesse processo é necessária à obtenção de mão de obra qualificada para trabalhar com esse novo sistema produtivo. Muitas vezes esta dificuldade acontece por conta da baixa escolaridade da maior parte dos trabalhadores rurais que se encontram no campo (COVA; FONTES, 2007).

Cabe ao produtor rural buscar qualificação para si e aos seus funcionários para que os mesmos consigam acompanhar a novas tecnologias e futuramente recrutar pessoas já qualificadas para o cargo, porém uma das principais dificuldades no campo é encontrar talentos capazes e interessados em permanecer no agronegócio, por conta disso a parte motivacional acaba se tornando imprescindível.

O presente trabalho teve como objetivo abordar os processos de recrutamento e seleção, treinamento e desenvolvimento, avaliação de desempenho, motivação e remuneração. Para isso, o artigo conta com uma revisão bibliográfica para atingir o objetivo proposto. 


\section{FUNDAMENTAÇÃO TEÓRICA}

As pessoas são importantes para as empresas independente do ramo de atividade, a escolha de abordar os processos de: recrutamento e seleção, treinamento e desenvolvimento, avaliação de desempenho, motivação e remuneração deve-se ao fato de que várias pesquisas anteriores apresentaram características sobre como a gestão de pessoas pode oferecer vantagem competitiva no setor agrícola.

\subsection{Recrutamento de pessoal}

O processo de recrutamento e seleção é considerado fundamental nas organizações, uma vez que sua tarefa é agregar funcionários capacitados. De acordo com Carvalho, Passos e Saraiva (2008), como as organizações são sistemas abertos, ou seja, com entradas e saídas de produtos e serviços, o processo de recrutamento e seleção deve ser adequado a fim de atingir seus objetivos.

O recrutamento é a fase inicial para o preenchimento de uma vaga em aberto, tornando-se um processo de identificação e atração de um grupo de candidatos, dentre os quais, posteriormente serão escolhidos alguns para serem contratados (FRANÇA, 2012; MILKOVICK; BOUDREAU, 2000). Existem duas formas de recrutamento, que são: interno, quando o requerente já trabalha na empresa e disponibiliza-se para trocar de função; e externo, quando se busca, no mercado do trabalho candidatos sem vínculos prévios com a empresa (FRANÇA, 2012).

Para que a organização tenha sucesso em seu recrutamento externo é importante utilizar as fontes disponíveis que são: banco de dados da empresa, indicações de colaboradores, cartazes internos e externos, entidades diversas. O processo de seleção tem se tornado cada vez mais elemento chave para as empresas, pois quanto maior o número de candidatos, mais seletivo será o processo de contratação.

Sob o mesmo aspecto, Hanashiro, Teixeira e Zacarelli (2008) destacam que se o recrutamento não for bem conduzido, há um aumento em falhas na escolha do melhor candidato a cada cargo. Nesse sentido, percebe-se como fundamental que os objetivos do recrutamento e seleção possam estar alinhados às políticas e práticas que identifiquem o modelo de gestão da organização a que se refere, levando em consideração os aspectos que 
revelam sua cultura, missão, visão e valores. Após o recrutamento, chega o momento de selecionar entre os candidatos o mais adequado para a vaga em aberto.

\subsection{Seleção de pessoal}

A seleção de pessoal tem como objetivo escolher os candidatos que se mostraram mais qualificados na apuração inicial do recrutamento. Com isso conclui-se que o recrutamento e a seleção são etapas de um mesmo processo (CARVALHO; NASCIMENTO, 2014).

O sistema de gestão de pessoas tem o desígnio de escolher uma metodologia específica, candidatos para o atendimento das necessidades internas da empresa (MARRAS,2011). Uma boa seleção é importante, por três razões:

- Para o bom desempenho da organização como um todo e a escolha das habilidades e atributos certos para o desenvolvimento sustentável.

- Porque há um gasto com os processos de recrutamento e seleção.

- Por eventuais problemas que a empresa pode sofrer com contratações negligentes (DESSLER, 2003).

Conclui-se que para se obter ganhos nesta atividade é preciso planejar, especificando a vaga, o perfil necessário para ocupá-la, o trabalho a ser feito, o tipo de experiência necessário, com quem o colaborador vai se relacionar, quem serão seus clientes internos e externos, e que tipo de valores o colaborador deve possuir (DESSLER, 2003).

Existem varias técnicas para a seleção de pessoas, que variam e se adequam com porte da organização, segmento, região de inserção, grau tecnológico da organização, produto fabricado, entre outros. De acordo com França (2012) as especificações e o planejamento para realização do processo de seleção se dividem em vários modelos e técnicas.

Quadro 1 - Técnicas utilizadas no processo de seleção

\begin{tabular}{|c|c|c|}
\hline & ETAPAS & OBJETIVO \\
\hline 1 & Entrevistas & $\begin{array}{r}\text { São utilizadas no processo seletivo e tem como objetivo conhecer o candidato e obter informações a } \\
\text { seu respeito. }\end{array}$ \\
\hline 2 & $\begin{array}{c}\text { Provas de } \\
\text { conhecimento }\end{array}$ & $\begin{array}{r}\text { Podem ser gerais quando buscam avaliar o grau de cultura geral do candidato ou específicas quando } \\
\text { visam avaliar os conhecimentos profissionais que são imprescindíveis para a vaga. }\end{array}$ \\
\hline 3 & $\begin{array}{c}\text { Testes } \\
\text { psicológicos }\end{array}$ & $\begin{array}{r}\text { Dividem-se em testes psicométricos que procuram medir aptidões individuais, testes de } \\
\text { personalidade que tem como objetivo identificar aspectos motivacionais, interesses e distúrbios. }\end{array}$ \\
\hline
\end{tabular}




\begin{tabular}{|c|c|c|c|c|c|}
\hline 4 & $\begin{array}{c}\text { Técnicas } \\
\text { vivenciais }\end{array}$ & $\begin{array}{c}\text { São situações criadas para os candidatos interagirem e participarem assim, seu comportamento é } \\
\text { avaliado em diferentes situações pré-definidas pelos avaliadores. Podem ser provas situacionais que } \\
\text { estão relacionadas às tarefas do cargo ou dinâmica de grupo. }\end{array}$ \\
\hline
\end{tabular}

Fonte: Elaborado pelos autores com base em França (2012).

Essa seleção por competências tem como base fatos reais e mensuráveis, como as capacidades, os atributos e as qualidades comportamentais (FLEURY, 2002). É importante ressaltar que o processo de seleção deve seguir os critérios da metodologia que será utilizada, buscando prevalecer-se a subjetividade e competência do selecionador e respeitar as características de cada candidato gerando maior produtividade para a organização (HANASHIRO, TEIXEIRA, ZACARELLI, 2008). Após a seleção e definição dos candidatos a organização tem como propósito a etapa de treinamento e desenvolvimento.

\subsection{Treinamento e desenvolvimento de pessoal}

Segundo Vizoli (2010), a fase de treinamento e desenvolvimento tem ganhado cada vez mais espaço nas empresas, além de aumentar a capacidade do colaborador, também estão a relacionados à motivação, que servem como forma de reconhecimento do valor dos funcionários para a organização e também da qualidade organizacional da empresa. Mesmo sendo uma das etapas de mais importância não existem muitos estudos relacionados ao treinamento e desenvolvimento no agronegócio, algo que se torna prejudicial por conta de funcionários que não estão devidamente instruídos.

O quadro 2 apresenta as principais diferenças entre treinamento e desenvolvimento.

Quadro 2 - Atributos e diferenças entre treinamento e desenvolvimento

\begin{tabular}{|c|c|c|}
\hline ATRIBUTOS & TREINAMENTO & DESENVOLVIMENTO \\
\hline OBJETIVO & $\begin{array}{c}\text { Suprir as necessidades de um funcionário para } \\
\text { o desempenho de seu cargo. }\end{array}$ & $\begin{array}{c}\text { Crescimento integral do colaborador, de maneira que ele } \\
\text { desenvolva-se ou atinja competências importantes } \\
\text { pessoais e para a organização. }\end{array}$ \\
\hline FUNÇÃO & $\begin{array}{c}\text { Corretiva: o colaborador deveria apresentar } \\
\text { determinadas competências neste momento, } \\
\text { porém não as apresenta. }\end{array}$ & $\begin{array}{c}\text { Preventiva: no futuro, será bom que o colaborador } \\
\text { apresente determinadas competências }\end{array}$ \\
\hline FOCO & No curto prazo. & No médio e longo prazo \\
\hline PROPOSITO & Voltado ao cargo. & Voltado a pessoa. \\
\hline
\end{tabular}




\begin{tabular}{|l|l|l|}
\hline INFLUÊNCIA & Específica e pontual & Holística e abrangente \\
\hline
\end{tabular}

Fonte: Elaborado pelos autores com base em Vizoli (2010).

É possível observar que existem algumas diferenças entre o treinamento e o desenvolvimento, e em relação às necessidades de treinamento, Robbins (2009) aponta como os principais fatores que influenciam um treinamento:

- Aquisição de novos maquinários ou introdução de processos que influenciem o cargo de um funcionário;

- Alteração de função, diminuição da produtividade ou qualidade dos serviços;

- Aumento do número de acidentes ou infração das normas de segurança do trabalho;

- Crescimento de reclamações de cliente e funcionários.

A partir dessas identificações, os tipos mais comuns de treinamento apresentados por Robbins (2009), Lacombe (2005) e Hanashiro, Teixeira e Zaccarelli (2008) são:

- Orientação inicial: nesta etapa se realiza quando um funcionário novo chega à empresa. Pode ser feito pelo chefe ou superior, tem como objetivo integrar o novo colaborador as normas e o funcionamento da empresa.

- Treinamento operacional: é considerado o treinamento essencial, pois, seu objetivo é aprimorar as aptidões técnicas dos funcionários.

- Treinamento gerencial: seu objetivo é preparar aqueles que exercem ou vão exercer alguma posição e chefia.

- Treinamento em serviço: é realizado com os funcionários no ambiente de trabalho, os equipamentos utilizados no dia a dia, e visa demonstrar a realidade enfrentada pelos colaboradores.

Esses são os tipos de treinamentos mais tradicionais de acordo com Robbins (2009), Lacombe (2005) e Hanashiro, Teixeira e Zaccarelli (2008). Robbins (2009) destaca que é possível individualizar o treinamento, adequando os conteúdos para cada funcionário de acordo com a sua necessidade, tornando assim mais eficaz à aprendizagem dos colaboradores. Os programas de treinamento e desenvolvimento são meios que melhoram o desempenho do funcionário tem grande influência na motivação dos mesmos. 


\subsection{Avaliações de desempenho de pessoal}

Através da avaliação de desempenho a empresa consegue identificar como os avaliados estão desenvolvendo as atividades que executam, essa etapa envolve as decisões dos funcionários na empresa, como as promoções e transferências de setor, isto é, algo que possibilita a identificação da necessidade de novos treinamentos (LAMCOBE, 2005; ROBBINS, 2009).

A gestão de desempenho é considerada um processo que busca proporcionar um ambiente de trabalho no qual os funcionários atinjam o melhor de suas habilidades (BOHLANDER; SNELL, 2015).

\subsection{Remuneração}

A remuneração para Bohlander e Snell (2015) fundamenta-se em três elementos: a remuneração direta, que é o salário do funcionário, incentivos, bonificações e comissões; a remuneração indireta, que envolve os outros benefícios oferecidos; e a recompensa não financeira, que inclui o reconhecimento, ambiente de trabalho e horário flexível, entre outros.

A remuneração deve ser administrada de forma que diminua os custos e que mantenham a motivação e o desempenho dos funcionários. De acordo com Vizoli (2010), a remuneração por um bom desempenho auxilia na produtividade e na redução dos custos de mão de obra, sem essa isso a motivação e o desempenho no ambiente de trabalho se torna baixa.

Porém, no setor agrícola o funcionário pode ser recompensado pela sua produção e ser pago de acordo com o que foi produzido. (YAMAUCHI; SANTINI PIGATTO; BAPTISTA, 2015). Desse conceito podemos ressaltar que a estratégia para a remuneração dos funcionários influencia, tanto na motivação quanto no nível de qualidade do serviço.

\section{PROCEDIMENTOS METODOLÓGICOS}

Para realização deste artigo foi realizada uma revisão bibliográfica a partir de materiais publicados em sites, revistas, cartilhas e artigos, com os objetivos específicos de 
identificar quais são as práticas de gestão de pessoas utilizadas pelos agricultores, recrutamento e seleção, treinamento e desenvolvimento motivação e remuneração.

\begin{abstract}
A pesquisa bibliográfica é feita a partir do levantamento de referências teóricas já analisadas, e publicadas por meios escritos e eletrônicos, como livros, artigos científicos, páginas de web sites. Qualquer trabalho científico inicia-se com uma pesquisa bibliográfica, que permite ao pesquisador conhecer o que já se estudou sobre o assunto. Existem, porém pesquisas científicas que se baseiam unicamente na pesquisa bibliográfica, procurando referências teóricas publicadas com o objetivo de recolher informações ou conhecimentos prévios sobre o problema a respeito do qual se procura a resposta (FONSECA, 2002, p. 32)
\end{abstract}

Segundo Gil (2007, p. 44), os exemplos mais característicos desse tipo de pesquisa são sobre investigações e ideologias ou aquelas que se propõem à análise das diversas posições acerca de um problema.

\title{
4 RESULTADOS E DISCUSSÃO
}

O setor do agronegócio tem sido responsável por aproximadamente $23 \%$ do Produto Interno Bruto (PIB) brasileiro dos últimos dez anos, ocupando importante posição na produção mundial de café, laranja, soja e cana-de-açúcar, além de se destacar na exportação mundial de carne bovina e de aves (CEPEA/ ESALQ, 2015; IBGE, 2016).

Segundo o Ministério da Agricultura, Pecuária e Abastecimento - MAPA (2015), as exportações advindas do agronegócio brasileiro alcançaram a cifra de US\$ 5,64 bilhões em janeiro de 2015.

As pessoas são importantes para as empresas independente do ramo de atividade, a escolha de abordar os processos de: recrutamento e seleção, treinamento e desenvolvimento, avaliação de desempenho, motivação e remuneração deve-se ao fato de que várias pesquisas anteriores apresentaram características sobre como a gestão de pessoas pode oferecer vantagem competitiva no setor agrícola.

Há uma grande importância de estudos relacionados ao agronegócio e gestão de pessoas, pois a demanda por alimentos em 2030, com a população mundial chegando a nove milhões de pessoas, será dois terços maiores que a atual. (GRANT, 2012). No Brasil um dos principais desafios é a renovação de mão-de-obra e a sucessão gerencial dos negócios, está cada vez mais difícil manter os jovens no campo, dessa forma, acredita-se que uma boa gestão pode ser uma ferramenta para amenizar tais problemas. 
Albuquerque e Amorin (2012) destacam a importância das organizações utilizarem metodologias para avaliação de desempenho por competências de seus colaboradores, a partir de uma pesquisa realizada em uma empresa de grande porte que atua na produção de frutas na região do Vale do São Francisco. Os autores reforçam a necessidade de se transformar competências individuais em competências organizacionais, através de uma gestão com foco em desenvolvimento de equipes eficientes.

Outra preocupação crescente refere-se às novas habilidades necessárias, pois os agricultores precisam aprender a lidar com tecnologias adicionais, gestão dos recursos, assunção de riscos, práticas financeiras e de proteção ambiental. Tais habilidades resultam do conhecimento e treinamento adquirido pelos produtores em sua trajetória, entretanto, nos países em desenvolvimento, a ascensão e difusão desses quesitos são muito remotas, já que não possuem uma política voltada para o acesso de informações e práticas dos pequenos produtores (GRANT, 2012; JÖHR,2012).

Santos et al (2018) destacam a importância da gestão de pessoas nas cooperativas agrícolas, e ressaltam a pesquisa de clima organizacional, como uma ferramenta capaz de medir o nível de relacionamento entre os funcionários e a empresa, e á partir destes resultados, implementar ações que possibilitem melhorar o ambiente de trabalho e consequentemente os resultados da organização.

\section{CONSIDERAÇÕES FINAIS}

A gestão de pessoas no setor agropecuário é essencial para a sobrevivência da organização, conhecer bem seu mercado e seus funcionários traz melhores resultados para a empresa. Estabelecer metas, medir os resultados e avaliar a eficiência dos processos, identificar novas oportunidades são ações que qualquer empreendedor rural precisa estar preparado para fazer.

Investir na equipe e ter um pessoal capacitado é a chave para obter o máximo retorno sobre os investimentos. Organizações de sucesso trabalham estabelecendo metas e monitorando os resultados, transformando dados em informação e agindo de maneira focada e efetiva. Gestão profissional significa crescimento, produtividade, qualidade e, consequentemente, lucratividade para todo o setor. 
Há vários desafios referentes à capacitação da gestão do setor agropecuário, principalmente nas propriedades rurais, onde muitas delas são carentes de sistemas de gestão, muitas vezes por conta da sucessão familiar e pela migração dos jovens para outras áreas.

Com base nisso os processos de recrutamento e seleção, treinamento e desenvolvimento, avaliação de desempenho, motivação e remuneração, é importante, além de instruir e motivar funcionários mais antigos atrair os jovens para o setor.

Durante todo o processo de contratação e capacitação é possível identificar o perfil de cada funcionário, assim o encaixando na vaga adequada a fim de se obter melhores resultados. Com as novas tecnologias chegando ao mercado, com o intuito de melhorar a produção o produtor ou proprietário tem que estar preparado com funcionários qualificados para atender essa demanda.

\section{REFERENCIAS}

ALBUQUERQUE, C. C, P.; AMORIN, T. N. G. F. Desempenho por Competências: um estudo de caso no âmbito de uma Empresa de Fruticultura utilizando o modelo de Leme. Revista Custos e @ gronegócio on line - v. 7, n. 3 - Set/Dez - 2011

BOHLANDER, G. W.; SNELL, S. A. Administração de Recursos Humanos. 16. ed. São Paulo: Cengage, 2015.

CARVALHO, A. V. de; NASCIMENTO, L. P. do. Administração de recursos humanos. 2. ed. São Paulo: Cengage Laerning, 2014.

CARVALHO, I. M. V; PASSOS, A. E. V. M.; SARAIVA, S. B. C. Recrutamento e Seleção por Competências. Rio de Janeiro: FGV, 2008.

CEPEA/ESALQ - Centro De Estudos Avançados Em Economia Aplicada Da Escola Superior De Agricultura "Luiz De Queiroz". 2015. Disponível em:

<http://www.cepea.esalq.usp.br/>.

COVAS, M.; FONTES, S.; Gestão de pessoas no agronegócio. Revista Convibra 2007 . Disponivel em<http://www.convibra.com.br/2007/congresso/artigos/316.pdf > . Acesso em 01 de set. 2019.

DESSLER, G. Administração de recursos humanos. Tradução Cecília Leão Oderich. Revisão técnica Irene Kazumi Miura. 2. ed. São Paulo: Prentice Hall, 2003.

DUTRA. J. S. Gestão de Pessoas: modelo, processos, tendências e perspectivas. São Paulo: Atlas, 2002 
FERRAZ, D. L. da S.; OLTRAMARI, A. P.; PONCHIROLLI, O. Gestão de Pessoas e Relações de Trabalho. São Paulo: Atlas, 2011.

FRANÇA, A. C. L. Práticas de Recursos Humanos- PRH: conceitos, ferramentas e procedimentos. 1. ed. São Paulo: Atlas, 2012.

GIL, A.C. Métodos e técnicas de pesquisa social. São Paulo: Atlas, 2007.

GRANT, H. et al. Farmers, Food and the Future: Take Action Now to Attract the Next Generation of Agricultural Leaders. International Food and Agribusiness Management Review, v. 15, n. A, 2012. Disponível em:

<https://ageconsearch.umn.edu/record/129167?n=en> . Acesso em: 10 set. 2019.

HANASHIRO, M. M.; TEIXEIRA, M. L. M.; ZACARELLI, L. M. Gestão do Fator

Humano: Uma visão baseada em stakeholders. 2. ed. São Paulo: Saraiva, 2008.

IBGE - Instituto Brasileiro De Geografia E Estatística. Disponível em:

$<$ http://www.ibge.gov.br/>.

JÖHR, H. Where are the Future Farmers to Grow Our Food. International Food and Agribusiness Management Review, v. 15, 2012. Disponível em: <

https://ageconsearch.umn.edu/record/129168?ln=en >. Acesso em: 8 de set. 2019

MAPA - Ministério Da Agricultura, Pecuária E Abastecimento. 2015. Disponível em: $<$ http://www.agricultura.gov.br/>.

MILKOVICH, G. T.; BOUDREAU, J. W. Administração de recursos humanos. Tradução Reynaldo C. Marcondes. São Paulo: Atlas, 2000.

MILKOVICH, G. T.; BOUDREAU, J. W. Administração de recursos humanos. Tradução Reynaldo C. Marcondes. São Paulo: Atlas, 2000.

ROBBINS, S. P. Administração: mudanças e perspectivas. São Paulo: Saraiva, 2009.

SANTOS, E. F. A. et al. CLIMA ORGANIZACIONAL: um estudo de caso em uma cooperativa do agronegócio. II Encontro Internacional de Gestão, Desenvolvimento e Inovação, 2018 - Naviraí - MS - Brasil

VIZOLI, M. Administração de recursos humanos. São Paulo: Academia Person, 2010.

YAMAUCHI, F.; PIGATTO, G. A. S.; BAPTISTA, R. D. Os fatores que influenciam no processo de adoção de inovação e os aspectos culturais: estudos de caso de produtores de amendoim do município de Tupã (SP). Revista ADMPG Gestão Estratégica, Ponta Grossa, v. 8, n. 2, p.45-53, 2015. Disponível em:<

http://www.admpg.com.br/revista2015_2/Artigos/Artigo5.pdf>. Acesso em: 05 set. 2019. 